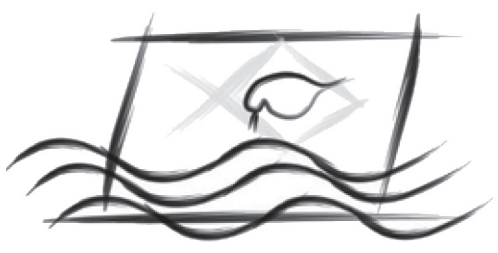

J. Braz. Soc. Ecotoxicol., v. 5, n. 1, 2010, 27-32

doi: $10.5132 /$ jbse.2010.01.005

ECOTOX - Brazil

\title{
Avaliação do Copépodo Acartia tonsa (Dana, 1849) como Organismo-Teste para Ensaios de Toxicidade Crônica
}

\author{
P. M. Ihara, G. L. L. Pinho* \& G. Fillmann \\ Laboratório de Microcontaminantes Orgânicos e Ecotoxicologia Aquática, Instituto de Oceanografia, \\ Universidade Federal do Rio Grande - FURG, Av. Itália, Km 8, CEP 96201-900, Rio Grande - RS, Brasil
}

(Received April 1, 2009; Accepted December 15, 2009)

\begin{abstract}
RESUMO
Atualmente, os ensaios ecotoxicológicos têm sido utilizados como uma importante ferramenta na avaliação da qualidade ambiental. Com objetivo de destacar o uso de organismos nativos em ensaios crônicos de curta duração, este estudo avaliou o potencial do copépodo Acartia tonsa em ensaios de reprodução. Ensaios semi-estáticos foram realizados expondo organismos adultos às concentrações de 0,$5 ; 0,75 ; 1,0 ; 1,5 ; 2,0$ e $2,5 \mathrm{mg} \cdot \mathrm{L}^{-1}$ de sulfato de zinco $\left(\mathrm{ZnSO}_{4} \cdot 7 \mathrm{H}_{2} \mathrm{O}\right)$. A produção de ovos por fềmea foi quantificada em 48 horas, obtendo a $\mathrm{CE}_{50}$ de $2,05 \mathrm{mg} \cdot \mathrm{L}^{-1}$ de sulfato de zinco (IC 95\% = 1,77 - 2,20 mg.L $\mathrm{L}^{-1}$ ). Ensaios agudos foram realizados concomitantemente, expondo os organismos as concentrações de 2,5;5,0; 7,5; 10;12,5 e 15 mg.L. $\mathrm{L}^{-1}$ de $\mathrm{ZnSO}_{4} \cdot 7 \mathrm{H}_{2} \mathrm{O}$. Em comparação aos efeitos agudos, o ensaio crônico obteve $\mathrm{CE}_{50}$ cerca de 1,9 vezes menor que a concentração letal $\left(\mathrm{CL}_{50}\right.$ de 3,80 mg.L-1-1). A resposta de sensibilidade foi cerca de 3 a 4 vezes menor que os demais ensaios reportados na literatura. Desta forma, estudos complementares são necessários para estabelecer as condições ótimas de exposição e verificar a possibilidade de efeitos subletais mais sensíveis.
\end{abstract}

Palavras-chave: Acartia tonsa, ensaio de toxicidade crônica, ecotoxicologia, produção de ovos, sulfato de zinco.

\section{ABSTRACT \\ Appraisal of Copepod Acartia tonsa (Dana, 1849) as a Chronic Toxicity Test Organism}

Ecotoxicological tests have been used as an important tool in assessing the environmental quality. In order to improve the use of marine and estuarine native organisms in chronic toxicity tests, this study evaluated the potential of the copepod Acartia tonsa in reproduction test. The sensitivity of $A$. tonsa was determined by exposing adults to $0.5,0.75,1.0,1.5,2.0$ and $2.5 \mathrm{mg}$. $\mathrm{L}^{-1}$ of zinc sulphate $\left(\mathrm{ZnSO}_{4} \cdot 7 \mathrm{H}_{2} \mathrm{O}\right)$. The production of eggs per female was measured after 48 hours of exposure, resulting in the $\mathrm{EC}_{50}$ of $2.05 \mathrm{mg} . \mathrm{L}^{-1}$ of zinc sulphate (CI $\left.95 \%=1.77-2.20 \mathrm{mg} . \mathrm{L}^{-1}\right)$. Acute toxicity test was also performed, exposing adults to $2.5,5.0,7.5,10,12.5$ and $15 \mathrm{mg} \cdot \mathrm{L}^{-1} \mathrm{ZnSO}_{4} \cdot 7 \mathrm{H}_{2} \mathrm{O}$. Compared to acute effects, the chronic test was 1.9 lower than the lethal concentration $\left(\mathrm{LC}_{50}\right.$ of $\left.3.80 \mathrm{mg} \cdot \mathrm{L}^{-1}\right)$. The sensitivity response of $A$. tonsa exposed to zinc presented an $\mathrm{EC}_{50} 3$ to 4 times higher than the others test organism described in the literature, so further studies are needed to improve sensitivity by developing and applying other sublethal endpoints.

Keywords: Acartia tonsa, chronic toxicity test, ecotoxicology, egg production, zinc sulfate. 


\section{INTRODUÇÃO}

Os organismos marinhos estão expostos a uma variedade de substâncias xenobióticas provenientes das mais diversas atividades antrópicas. A exposição aos contaminantes pode ocasionar a mortalidade de diversos organismos, além de causar alterações de comportamento, reprodução e inibições de crescimento. Estas alterações podem danificar a estrutura da população e, por conseqüência, resultar em potenciais impactos nas interações de diferentes cadeias alimentares (Hoffmann et al., 1995).

Atualmente, a manutenção da qualidade dos ambientes aquáticos em relação ao lançamento de efluentes e produtos químicos industriais é prevista na legislação brasileira. De acordo com a Resolução n 357 (CONAMA, 2005), eventuais interações entre substâncias, especificadas ou não na legislação, não poderão conferir às águas características de causar efeitos letais ou subletais. Desta forma, para que seja eficientemente aplicada, ensaios ecotoxicológicos têm sido exigidos para determinar a toxicidade de efluentes e de compostos químicos lançados no ambiente aquático (Araújo \& Nascimento, 1999).

No Brasil, a ecotoxicologia marinha é relativamente recente de forma que são poucas as espécies nativas que apresentam ensaios ecotoxicológicos padronizados e protocolados. Para ambientes marinhos e estuarinos, apenas as espécies de ouriço-do-mar Lytechinus variegatus e Echinometra lucunter, os misidáceos Mysidopsis juniae e Mysidium gracile e a bactéria Vibrio fisheri apresentam ensaios ecotoxicológicos normalizados pela Associação Brasileira de Normas Técnicas (ABNT 2005, 2006a,b).

Em geral, os ensaios para avaliação da toxicidade aguda são realizados através de kits comerciais do tipo Microtox ( $V$. fischeri) ou através de organismos obtidos de cultivo (M. juniae e M. gracile) (ABNT 2005, 2006a). Já os ensaios de toxicidade crônica são realizados com ouriços-do-mar coletados diretamente do ambiente e, portanto, restrito aos locais onde há a presença destas espécies (ABNT, 2006b). Devido à limitação regional com que as espécies normalizadas são encontradas no ambiente, ensaios crônicos utilizando organismos alternativos têm sido testados (Laitano et al., 2008; Jorge \& Moreira, 2005). No entanto, nem todos apresentam os critérios necessários para serem utilizados rotineiramente como organismo-teste, tais como: curto ciclo de vida, facilidade de cultivo e/ou representatividade ecológica.

Deste modo, tendo em vista a necessidade de ensaios crônicos com organismos marinhos e estuarinos nativos, este trabalho teve o objetivo de avaliar o uso do copépodo planctônico Acartia tonsa em ensaios de reprodução. A. tonsa é uma espécie recomendada por órgãos internacionais (ex. International Organization for Standardization) para ensaios de toxicidade aguda e que apresenta requisitos necessários para a utilização em ensaios de toxicidade crônica. Considerando que os ensaios ecotoxicológicos devem ser realizados preferencialmente com espécies sensíveis, a aplicação da $A$. tonsa em ensaios crônicos foi avaliada com base na sensibilidade do organismo frente à substância de referência sulfato de zinco. $\mathrm{O}$ intuito deste trabalho foi avaliar o uso da $A$. tonsa como organismo-teste, visando com isto ampliar a opção de organismos disponíveis para a realização de ensaios de toxicidade no Brasil.

\section{MATERIAL E MÉTODOS}

\section{Cultivo do Copépodo}

Os ensaios de toxicidade com Acartia tonsa foram realizados com organismos fornecidos pelo Laboratório de Zooplâncton da Universidade Federal do Rio Grande - FURG. Os organismos foram mantidos em sala climatizada a $24{ }^{\circ} \mathrm{C}$, fotoperíodo de $12 \mathrm{C}: 12 \mathrm{E}$ horas e aeração constante. Os cultivos foram mantidos em água marinha filtrada ( $1 \mu \mathrm{m}$, salinidade 30$)$ e com fornecimento diário de uma dieta mista de microalgas (Thalassiosira weissflogii e Isochrysis galbana), conforme Kaminski (2004). A renovação de água do cultivo foi realizada semanalmente com auxílio de redes de malhas de $300 \mu \mathrm{m}$ (coleta de adultos) e de $45 \mu \mathrm{m}$ (coleta de ovos e náuplios).

\section{Produção de fitoplâncton}

Cultivos de Thalassiosira weissflogii e Isochrysis galbana foram iniciados para fornecer suporte na provisão diária de alimento ao cultivo de $A$. tonsa e durante os ensaios de toxicidade crônica. Os inóculos foram fornecidos pelo Laboratório de Ecologia de Fitoplâncton e de Microorganismos Marinhos (FURG). Cultivos foram mantidos em erlenmeyers de $100 \mathrm{~mL}$, a $20^{\circ} \mathrm{C}$, iluminação entre 800 e 1000 lux, fotoperíodo e aeração constante. Semanalmente foram re-inoculados em galões de $3 \mathrm{~L}$ contendo água do mar filtrada $(1 \mu \mathrm{m})$ em meio de cultivo F/2 (Guillard, 1975).

\section{Seleção dos organismos}

Para os ensaios de toxicidade foram utilizados organismos previamente selecionados de acordo com descrição de cada metodologia. Nos ensaios de toxicidade crônica foram utilizados copépodos adultos obtidos do cultivo, com idade entre 30 e 40 dias. Antes do início dos ensaios ( 24 horas), machos e fêmeas foram separados em lupa com pipetas Pasteur e colocados em recipientes distintos. A separação foi realizada para minimizar a possibilidade de que os ovos quantificados durante os ensaios fossem resultantes de uma copulação anterior. Os ensaios de toxicidade aguda foram realizados com organismos adultos, sem distinção de sexo, conforme ISO 14669, (ISO, 1999).

\section{Ensaios de toxicidade aguda com Acartia tonsa}

Os ensaios de toxicidade aguda com o copépodo foram realizados com base na metodologia protocolada para $A$. tonsa (ISO, 1999). Foram utilizados organismos adultos em ensaios estáticos de 48 horas, sem fornecimento de alimentação. Os ensaios foram realizados em frascos de polietileno descartáveis com $100 \mathrm{~mL}$ de solução-teste e mantidos sob as mesmas condições de cultivo, exceto aeração. Foram utilizadas soluções nas concentrações de 2,$5 ; 5,0 ; 7,5 ; 10 ; 12,5$ e 15 mg. $L^{-1}$ de $\mathrm{ZnSO}_{4} \cdot 7 \mathrm{H}_{2} \mathrm{O}(\mathrm{SYNTH})$. As concentrações foram realizadas em triplicata, utilizando 20 organismos por réplica. Os organismos vivos e mortos foram quantificados em 24 e 48 horas. A mortalidade foi constatada pela ausência de movimento, mesmo após estímulo físico, sendo confirmada através de observações em lupa. Os valores de $\mathrm{CL}_{50}$ e os intervalos de confiança de $95 \%$ (IC 95\%) foram obtidos pelo programa estatístico Trimmed Spearman-Karber (Hamilton et al., 1977). 


\section{Ensaios de toxicidade crônica com Acartia tonsa}

Os ensaios de toxicidade crônica foram realizados visando verificar alterações no potencial reprodutivo do copépodo Acartia tonsa após 48 horas de exposição. Foram realizados ensaios semi-estáticos, com renovação da solução-teste em 24 horas. Os ensaios foram realizados em tubos de ensaio contendo $3 \mathrm{~mL}$ de solução-teste e um casal de organismos previamente separado. As soluções foram preparadas com água marinha filtrada $(1 \mu \mathrm{m}$, salinidade 30) e sulfato de zinco heptahidratado (SYNTH) nas concentrações de 0,$5 ; 0,75 ; 1,0 ; 1,5 ; 2,0$ e 2,5 mg.L - $^{-1}$ de $\mathrm{ZnSO}_{4} \cdot 7 \mathrm{H}_{2} \mathrm{O}$. Foram utilizadas 12 réplicas em cada concentração. Os ensaios foram realizados com fornecimento de alimento (dieta mista de microalgas) sob as mesmas condições de cultivo, exceto aeração. A alimentação foi dada ad libitum, fornecendo a mesma concentração de microalgas para todas as réplicas, de modo a evitar que a produção de ovos fosse limitada pelo fornecimento de alimento. Os ensaios foram realizados em constante agitação (mesa agitadora) para prevenir que a disponibilidade do alimento fosse afetada pela decantação das microalgas. Os copépodos vivos, mortos, ovos e náuplios foram quantificados em 24 e 48 horas. Como produção de ovos, considerou-se o somatório total de ovos e náuplios quantificado durante 48 horas de experimento. Para obtenção da $\mathrm{CE}_{50}$, referente a concentração efetiva mediana dos organismos expostos, foi utilizado a produção média de cada concentração, considerando-se apenas as réplicas em que os organismos mantiveram-se vivos até a finalização dos ensaios. Os valores de $\mathrm{CE}_{50} 48$ horas e os intervalos de confiança de $95 \%$ foram obtidos por interpolação linear através do programa estatístico ICp, versão 2.0 (EPA, 1993). Diferenças significativas $(p<0,05)$ de efeito em relação ao Controle foram avaliadas por Análise de Variância (ANOVA), seguido por teste de Tukey a posteriori.

\section{RESULTADOS}

\section{Ensaio de toxicidade aguda}

Uma clara relação dose-resposta foi observada já que houve um aumento da mortalidade de Acartia tonsa com o aumento das concentrações de exposição ao sulfato de zinco (Figura 1). As mortalidades observadas no Controle estiveram acima de $80 \%$ de sobrevivência. Efeitos foram observados em 24 e 48 horas, obtendo-se a CL $\mathrm{CL}_{50}$ de 3,80 mg.L $\mathrm{L}^{-1}$ de sulfato de zinco (IC 95\%= $3,39-4,26 ; n=10)$ ao final de 48 horas de exposição.

\section{Ensaio de toxicidade crônica}

A produção de ovos por fêmea de $A$. tonsa diminuiu com o aumento das concentrações ao final de 48 ( 0 - 48 horas) horas de exposição (Figura 2). Diferença significativa $(\mathrm{p}<0,05) \mathrm{em}$ relação ao Controle foi observado somente na concentração de 2,5 mg. $\mathrm{L}^{-1}$ de sulfato de zinco. A produção média de ovos no Controle foi de $69,8( \pm 12,9)$ ovos fêmea ${ }^{-1}$ ao final de 48 horas, enquanto que na maior concentração de exposição $\left(2,5 \mathrm{mg} \cdot \mathrm{L}^{-1}\right.$ de sulfato de zinco) a produção foi de $9,0( \pm 5,6)$ ovos fêmea $^{-1}$ (Figura 2). A concentração efetiva $\left(\mathrm{CE}_{50}\right)$ referente à toxicidade crônica, determinada a partir da produção média total obtida ao final de 48 horas de experimento, foi de $2,05 \mathrm{mg} . \mathrm{L}^{-1}$ de sulfato de zinco (IC 95\% = 1,77 - 2,20 mg.L $\mathrm{L}^{-1}$ ).

\section{DISCUSSÃO}

O copépodo Acartia tonsa é uma espécie recomendada por órgãos internacionais para ensaios de toxicidade aguda (ISO 14669). Por consequência, tem sido mundialmente utilizado na avaliação da toxicidade de compostos presentes no ambiente marinho (Sverdrup et al., 2002; Christoffersen et al., 2003; Tsui \& Chu, 2003; Willis \& Ling, 2003). No Brasil, o uso da $A$. tonsa tem sido aplicado principalmente em estudos fisiológicos, na avaliação de efeitos causados por metais como cobre e prata (Pedroso et al., 2007; Pinho et al., 2007), sendo somente recentemente utilizado na determinação da toxicidade de compostos específicos, como derivados de petróleo, metais e detergentes (Rossato, 2008; Ávila et al., 2010).

No presente estudo, a exposição do copépodo $A$. tons $a$ ao zinco em ensaios de toxicidade aguda apresentou uma sensibilidade similar ao reportado ao copépodo Acartia lilljeborgi (Nipper, 1993). Comparado aos ensaios com organismos de diferentes grupos taxonômicos, a sensibilidade da $A$. tonsa ao zinco foi cerca de 3 vezes menor que a reportada para o misidáceo (Mysidopsis juniae) e 4 vezes menor que ao cirripédio Megabalanus coccopoma (Prósperi et al., 1994; Souza et al., 2007) (Tabela 1).

No ensaio crônico de reprodução, $A$. tonsa também apresentou uma menor sensibilidade ao zinco quando comparado aos demais ensaios de toxicidade crônica descritos na literatura (Tabela 2). A sensibilidade da $A$. tonsa foi cerca de 3 vezes

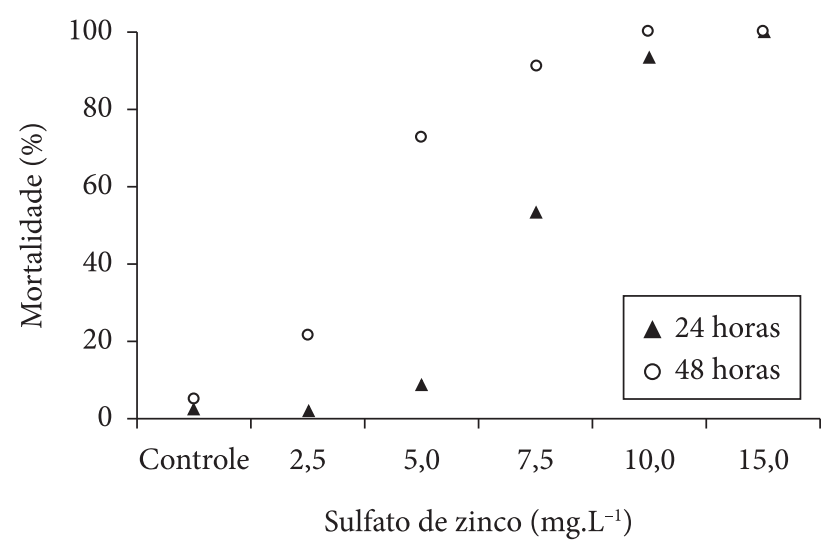

Figura 1 - Porcentagem de mortalidade de Acartia tonsa ao final de 24 e 48 horas de exposição ao sulfato de zinco $(\mathrm{n}=10)$.

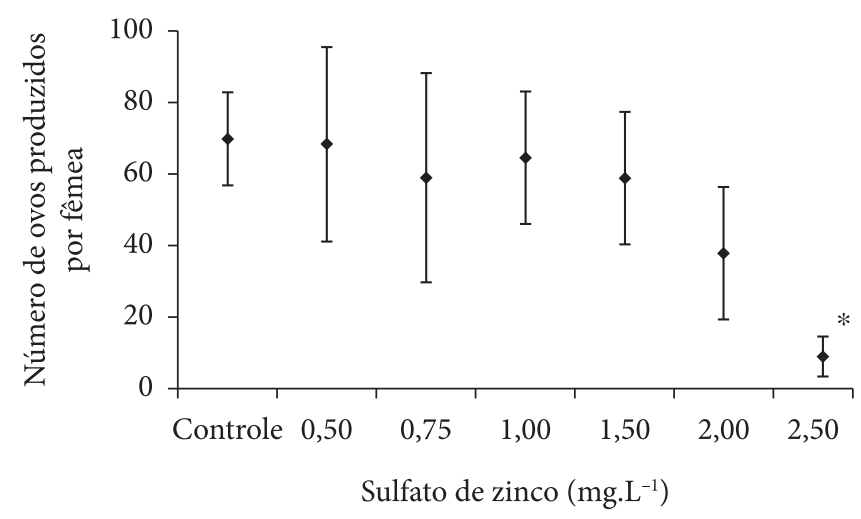

Figura 2 - Produção total de ovos por fêmea (média \pm DP; $\mathrm{n}=12$ ) de Acartia tonsa ao final de 48 horas de exposição ao sulfato de zinco. * Diferença significativa em relação ao Controle $(\mathrm{p}<0,05)$. 
menor que a descrita ao ouriço-do-mar $L$. variegatus, espécie padronizada pela $A B N T$, e cerca de 10 vezes menor que a sensibilidade reportada para Arbacia lixula (Prósperi et al., 1994; Jorge \& Moreira, 2005; Gimiliane et al., 2008). Cabe destacar, no entanto, que os ensaios crônicos com ouriçosdo-mar consistem em avaliar efeitos embrio-larvais de curta duração, no qual o efeito tóxico observado é desenvolvimento anormal ou retardado de larvas pluteus (Prósperi et al., 1994; Jorge \& Moreira, 2005; Gimiliane et al., 2008). No presente estudo, entretanto, o ensaio de toxicidade crônica foi baseado em ensaios de reprodução, onde o efeito tóxico observado é a redução do potencial reprodutivo da $A$. tonsa.

A redução do número de ovos produzidos por fêmea, no ambiente aquático, representa um importante efeito biológico e ecológico. Para os copépodos, o recrutamento populacional e as taxas de natalidade correspondem a uma resposta direta do potencial reprodutivo de cada organismo (Uye \& Shibuno, 1992; Hirst \& Mc Kinnon, 2001). Algumas espécies, como A. tonsa, apresentam produção contínua de ovos, que tem início logo após a maturação sexual e que se prolonga até o final do ciclo de vida (Kiørboe et al., 1985). A produção de ovos e larvas no ambiente apresenta uma grande influência ecológica para as espécies pelágicas ocorrentes, contribuindo, desta maneira, para o sucesso de muitas espécies marinhas (Runge, 1988).

No presente estudo, a produção de ovos em laboratório obteve no Controle uma produção diária média de $35( \pm 11)$ ovos por fêmea, semelhante à produção de cultivo reportada por Medina \& Barata (2004) em torno de 25 a 50 ovos fêmea ${ }^{-1}$ dia $^{-1}$. Nos ensaios de toxicidade, a avaliação da produção de ovos de $A$. tonsa tem a vantagem desta espécie não carregar sacos ovígeros, liberando-os diretamente na coluna de água (Dussart \& Defaye, 1995). Desta forma, a redução da produção de ovos é um efeito rapidamente observado.

Para Støttrup \& Jensen (1990), a produção de ovos da $A$. tonsa está intimamente associada à dieta alimentar. Por ser uma espécie oportunista, esta não acumula reservas de energia, de forma que a produção decresce rapidamente quando as condições alimentares encontram-se deterioradas (Kiørboe et al., 1985). Por este motivo, para que a redução do potencial reprodutivo seja uma resposta detectada em ensaios de toxicidade, estes devem ser realizados preferencialmente com fornecimento de alimento. Desta forma, a redução do potencial reprodutivo da $A$. tonsa pode estar relacionada, no presente estudo, a dois processos de exposição: o contato direto do organismo com o contaminante dissolvido na água e/ou o consumo de partículas de alimento contendo o contaminante adsorvido e/ou acumulado. Os dados obtidos, no entanto, não permitem determinar a exata via de assimilação do contaminante. Hook \& Fisher (2000), entretanto, avaliando o efeito da prata sobre a reprodução de $A$. tonsa, constataram que a exposição de Ag através do fitoplâncton apresentou $75 \%$ a mais de efeito quando comparado apenas à fração dissolvida, indicando a alimentação ser uma importante rota de exposição.

Considerando que o objetivo deste trabalho foi avaliar o uso da $A$. tonsa em ensaios de toxicidade crônica, é importante analisar de uma forma integrada e concomitante os resultados obtidos nos ensaios crônicos e agudos. Observa-se no ensaio crônico $\left(\mathrm{CE}_{50}=0,48 \mathrm{mg} \cdot \mathrm{L}^{-1} \mathrm{Zn}\right)$ que o valor nominal da $\mathrm{CE}_{50}$ foi cerca de 1,9 vezes menor que a $\mathrm{CL}_{50}\left(0,91 \mathrm{mg} \cdot \mathrm{L}^{-1} \mathrm{Zn}\right)$. Isso indica que, embora o ensaio crônico de reprodução de 48 horas com $A$. tonsa tenha se mostrado viável para detectar efeitos crônicos, representa um pequeno ganho na sensibilidade do efeito em relação aos ensaios agudos $\left(\mathrm{CE}_{50}<2\right.$ vezes a $\left.\mathrm{CL}_{50}\right)$. Estudos utilizando um mesmo organismo para ensaios de toxicidade crônica e aguda são descritos na literatura e, assim como observado, nem todos apresentam uma diferença relevante de sensibilidade entre efeitos crônicos e agudos. Em Souza et al. (2007), efeitos letais e subletais com larvas de Megabalanus coccopoma expostos ao sulfato de zinco demonstraram concentrações similares na avaliação das respostas de mortalidade $\left(\mathrm{CL}_{50} 48\right.$ horas de 0,99 mg. $\left.\mathrm{L}^{-1}\right)$ e mobilidade $\left(\mathrm{CE}_{50} 48\right.$ horas de 1,06 mg.L $\left.\mathrm{L}^{-1}\right)$. De acordo com os autores, os resultados foram atribuídos ao tipo de efeito subletal considerado e as condições de execução dos ensaios de toxicidade. Além disso, Christoffersen et al. (2003), utilizando o lauril alquil sulfonado (LAS) como substância de referência, constataram resultados semelhantes aos obtidos no presente estudo para $A$. tonsa. Os efeitos letais e subletais $\left(\mathrm{CL}_{50}\right.$ e $\mathrm{CE}_{50}$ ) para sobrevivência e produção de ovos resultaram, respectivamente, em valores de 1,11 e $0,92 \mathrm{mg} \cdot \mathrm{L}^{-1}$ de LAS, o que corresponde a uma $\mathrm{CE}_{50} 1,6$ vezes menor que a $\mathrm{CL}_{50}$ para o mesmo período de exposição (48 horas).

Tabela 1 - Valores de $\mathrm{CL}_{50}$ para diferentes organismos expostos ao zinco (mg. $\left.\mathrm{L}^{-1}\right)$ em ensaios de toxicidade aguda.

\begin{tabular}{lcccc}
\hline \multicolumn{1}{c}{ Organismo-teste } & $\mathrm{CL}_{50}$ & Zn $\left(\mathrm{mg} \cdot \mathrm{L}^{-1}\right)$ & $\mathrm{IC}(95 \%)$ & Referência bibliográfica \\
\hline Mysidopsis juniae & 96 horas & 0,28 & $0,22-0,32$ & Prósperi et al. (1994) \\
Megabalanus coccopoma & 48 horas & 0,22 & $0,06-0,44$ & Souza et al. (2007) \\
Acartia lilljeborgi & 48 horas & 0,89 & - & Nipper (1993) \\
Acartia tonsa & 48 horas & 0,86 & $0,77-0,96$ & Presente trabalho \\
\hline
\end{tabular}

Tabela 2 - Valores de $\mathrm{CE}_{50}$ para diferentes organismos expostos ao zinco (mg. $\left.\mathrm{L}^{-1}\right)$ em ensaios de toxicidade crônica.

\begin{tabular}{lcccc}
\hline Organismo-teste & $\mathrm{CE}_{50}$ & $\mathrm{Zn}\left(\mathrm{mg} \cdot \mathrm{L}^{-1}\right)$ & $\mathrm{IC}(95 \%)$ & Referência bibliográfica \\
\hline Arbacia lixula & 24 horas & 0,048 & $0,03-0,06$ & Prósperi et al. (1994) \\
Lytechinus variegatus & 24 horas & 0,150 & $0,10-0,20$ & Gimiliani et al. (2008) \\
Perna perna & 48 horas & 0,104 & $0,07-0,13$ & Jorge \& Moreira (2005) \\
Acartia tonsa & 48 horas & 0,479 & $0,41-0,51$ & Presente trabalho \\
\hline
\end{tabular}


A resposta de sensibilidade observada nos ensaios crônicos $\operatorname{com} A$. tonsa está provavelmente relacionada à metodologia de exposição e ao caráter oportunista da espécie, que faz com que a utilização do copépodo no ensaio de reprodução não seja um indicador de efeito subletal particularmente sensível em comparação ao ensaio agudo. Dentre os principais fatores responsáveis por influenciar a sensibilidade da espécie no ensaio crônico, destacam-se o tempo de duração do ensaio (48 horas), a influência da alimentação (dieta mista de microalgas) e a alta variabilidade entre as réplicas, de forma que são ainda necessários estudos metodológicos mais detalhados a fim de otimizar estes ensaios.

No que diz respeito à sensibilidade, ainda que $A$. tonsa tenha se apresentado menos sensível ao zinco em relação às demais espécies reportadas na literatura, esta espécie ainda possui um elevado potencial de aplicação em ensaios ecotoxicológicos. Ao contrário das espécies padronizadas pela Associação Brasileira de Normas Técnica (ABNT) para ensaios de toxicidade crônica, o copépodo $A$. tonsa apresenta uma metodologia de cultivo previamente estabelecida (Medina \& Barata, 2004) e de relativamente fácil manutenção. A utilização de espécies com capacidade de cultivo é extremamente importante para ensaios de toxicidade crônica, pois possibilita a disponibilidade contínua de organismos e a realização de ensaios com organismos adaptados a condições constantes. Estas condições são necessárias, principalmente para os ensaios crônicos, onde os efeitos subletais são avaliados com menor possibilidade de interferentes.

Nos últimos anos, ressalta-se que vem se observando no cenário nacional uma redução drástica nas populações de Lytechinus variegatus, espécie padronizada pela ABNT para ensaios de toxicidade crônica. Isto ocorre principalmente ao longo da costa Nordeste do Brasil, o que vem restringindo a sua utilização em ensaios ecotoxicológicos. Do mesmo modo, nas regiões temperadas, a abundância de $L$. variegatus diminui nos meses de inverno, em decorrência da redução da temperatura da água (Lotufo, 2008). Dentro deste contexto, a aplicação de ensaios ecotoxicológicos com $A$. tonsa pode se tornar uma opção viável para determinação de efeitos crônicos. No entanto, estudos com outras substâncias são necessários para uma completa avaliação da sensibilidade da espécie quanto a efeitos subletais. Além disso, estudos complementares são também imprescindíveis para melhorar a sensibilidade dos ensaios através do desenvolvimento e aplicação de outras respostas subletais, assim como no estabelecimento das condições ótimas de exposição ao organismo.

\section{REFERÊNCIAS BIBLIOGRÁFICAS}

ABNT - Associação Brasileira de Normas Técnicas, 2005, NBR 15308, Ecotoxicologia Aquática: Toxicidade aguda - métodos de ensaio com misidáceos (Crustacea). Rio de Janeiro, 17p.

ABNT - Associação Brasileira de Normas Técnicas, 2006a, NBR 15411, Ecotoxicologia Aquática: Determinação do efeito inibitório de amostras de água sobre a emissão de luz de Vibrio fisheri (Ensaio de bactéria luminescente). Rio de Janeiro, (Parte 1, 2, 3), p. 14-15, 18.

ABNT - Associação Brasileira de Normas Técnicas, 2006b, NBR 15350, Ecotoxicologia Aquática: Toxicidade crônica de curta duração: método de ensaio com ouriço-do-mar (Echinodermata: Echinoidea). Rio de Janeiro, 17p.

ARAÚJO, M. M. S. \& NASCIMENTO, I. A., 1999, Testes ecotoxicológicos marinhos: Análise de sensibilidade. Ecotoxicol. Environ. Restor., 2:41-47.

ÁVILA, T. R., BERSANO, J. G. F. \& FILLMANN, G., 2010, Lethal and sub-lethal effects of the water-soluble fraction of a light crude oil on the planktonic copepod Acartia tonsa. J. Braz. Soc. Ecotoxicol., 5: 19-25. doi: 10,5132/jbse.2010.01.004

CHRISTOFFERSEN, K., HANSEN, B. W., JOHANSSON, L. S. \& KROG, E., 2003, Influence of LAS on marine calanoid copepod populations dynamics and potential reproduction. Aquat. Toxicol., 63:405-416. doi: 10.1016/S0166-445X(02)00200-X

CONAMA-Conselho Nacional do meio Ambiente, 2005, Resolução CONAMA $n^{\circ} 357$, de 11 de março de 2005. Available from: www. mma.org.br

DUSSART, B. H. \& DEFAYE, D., 1995, Copepoda. Introduction to the Copepoda. Guides to the identification of the microinvertebrates of the continental waters of the world. SPB Academic Publishing, Amsterdan, 277p.

EPA - U.S. Environmental Protection Agency, 1993, A linear Interpolation method for sublethal toxicity: The inhibition concentration (ICp) Approach. Versão 2.0. Environmental Research Laboratory, Duluth, MN.

GIMILIANE, G. T., SOARES, K. S. B., VILELA, L. Q., CORTEZ, F. S., CESAR, A., SANTOS, A. R. \& PEREIRA, C. D. S., 2008, Taxa de fecundação e desenvolvimento embriolarval de Lytechinus variegatus (Echinodermata: Echinoidea) expostos ao zinco. Anais do X Congresso Brasileiro de Ecotoxicologia, Bento Gonçalves, RS, p. 177.

GUILLARD, R. R. L., 1975, Culture of phytoplankton for feeding marine invertebrate animals. In: SMITH, W. L., CHANLEY, M. H. (Eds.). Culture of marine invertebrate animals. New York, Plenum Press, p. 29-60.

HAMILTON, M. A., RUSSO, R. C. \& THURSTON, R. V., 1977, Trimmed Spearman-Karber method for estimating median lethal concentrations in toxicity bioassays. Environ. Sci. Technol., 11:714-719.

HIRST, A. G. \& McKINNON, A. D., 2001, Does egg production represent adult female copepod growth? A call to account for body weigth changes. Mar. Ecol. Prog. Ser., 223:179-199.

HOFFMANN, D. J., RATTNER, B. A., BURTON, A. A. \& CAIRNS Jr., F., 1995, Handbook of ecotoxicology, Lewis Publishers (Eds.). CRC Press, Boca Raton, Florida.

HOOK, S. E. \& FISHER, N. S., 2000, Exposure to silver via food reduces egg production and viability in herbivorous copepods and cladocerans. Mar. Environ. Res., 50:551-552. doi: 10.1016/ S0141-1136(00)00250-6

ISO - International Organization for Standardization, 1999, ISO 14669:1999, Water Quality - Determination of acute lethal toxicity to marine copepods (Copepoda, Crustacea). Geneva.

JORGE, R. A. D. L. V. C. \& MOREIRA, G. S., 2005, Use of dodecyl sulfate and zinc sulfate as reference substances for toxicity tests with the mussel Perna perna (Linnaeus, 1758) (Mollusca: Bivalvia). Ecotoxicol. Environ. Saf., 61:280-285. doi: 10.1016/j. ecoenv.2004.09.005

KAMINSKI, S. M., 2004, Influência da alimentação sobre a reprodução e o desenvolvimento do copépodo calanoida Acartia 
tonsa DANA, 1984, em cultivo intensivo. Dissertação (Mestrado em Aqüicultura), Universidade Federal de Santa Catarina (UFSC), Santa Catarina, 66 p.

KIØRBOE, T., MØHLENBERG, F. \& HAMBURGER, K., 1985, Bioenergetic of the planktonics copepod Acartia tonsa: relation between feeding, eggs production and respiration, and composition of specific dynamic action. Mar. Ecol. Prog. Ser., 26:85-97.

LAITANO, K. S., GONÇALVES, C. \& RESGALLA Jr., C., 2008, Viabilidade do uso da bolacha-do-mar Mellita quinquiesperforata como organismo teste. J. Braz. Soc. Ecotoxicol., 3:9-14. doi: $10,5132 /$ jbse. 2008.01 .002

LOTUFO, L.V., 2008, Echinodermata como modelo para ecotoxicologia. XXVII Congresso Brasileiro de Zoologia, Curitiba, PR.

MEDINA, M. \& BARATA, C. 2004, Static-renewal culture of Acartia tonsa (Copepoda: Calanoida) for ecotoxicological testing. Aquaculture, 229:203-213. doi: 10.1016/S0044-8486(03)00389-2

NIPPER, M. G., BADARÓ-PEDROSO, C., JOSÉ, V. F. \& MELO, S. I. R., 1993, Toxicity testing with coastal species of southeastern Brazil: Mysis and copepods. Bull. Environ. Contam. Toxicol., 51:99-106. doi: 10.1007/BF00201007

PEDROSO, M. S., PINHO, G. L. L., RODRIGUES, S. C. \& BIANCHINI, A., 2007, Mechanism of acute silver toxicity in the euryhaline copepod Acartia tonsa. Aquat. Toxicol., 82:173-180. doi: 10.1016/j.aquatox.2007.02.009

PINHO, G. L. L., PEDROSO, M. S., RODRIGUES, S. C., SOUZA, S. S. \& BIANCHINI, A., 2007, Physiological effects of copper in the euryhaline copepod Acartia tonsa: Waterborne versus waterborne plus dietborne exposure. Aquat. Toxicol., 84:62-70. doi: 10.1016/j.aquatox.2007.06.001

PRÓSPERI, V. A., NIPPER, M. G. \& VALERIA, S., 1994, Desenvolvimento de metodologia de cultivo e testes de toxicidade com organismos marinhos. In: Relatório Técnico da Companhia de Tecnologia de Saneamento Ambiental (SP). CETESB: 028755028756 .

ROSSATO, M. 2008. Avaliação do copépode Acartia tonsa como organismo-teste em ensaios ecotoxicológicos: Comparação entre diferentes espécies utilizadas no Brasil. Monografia (Graduação), Curso de Oceanologia, Universidade Federal do Rio Grande, Rio Grande, RS, 62 p.

RUNGE, J. A., 1988, Should we expect a relationship between primary production and fisheries? The role of copepod dynamics as filter of trophic variability. Hydrobiologia, 167-168:61-71.

SOUZA, R. C., POLEZA, M., MÁXIMO, M. V. \& RESGALlA Jr., C., 2007, Utilização de larvas do cirripedia Megabalanus coccopoma (Darwin, 1854) como organismo-teste na avaliação de toxicidade aguda. J. Braz. Soc. Ecotoxicol., 2:7-14. doi: 10,5132/jbse.2007.01.002

STØTTRUP, J. G. \& JENSEN, J., 1990, Influence of algal diet on feeding and egg-production of the calanoid Acartia tonsa DANA. J. Exp. Mar. Biol. Ecol., 141:87-105. doi: 10.1016/00220981(90)90216-Y

SVERDRUP, L. E., CHARLOTTE, S. F., WEIDEBORG, M., VIK, E. A. \& STENERSEN, J., 2002, Relative sensitivity of one freshwater and two marine acute toxicity tests as determined by testing 30 offshore E\&P chemicals. Chemosphere, 46:311-318. doi: 10.1016/S0045-6535(01)00123-0

TSUI, M. T. K. \& CHU, L. M., 2003, Aquatic toxicity of glyphosate based formulations: comparison between different organisms and the effects of environmental factors. Chemosphere, 52:11891197. doi: 10.1016/S0045-6535(03)00306-0

UYE, S. \& SHIBUNO, N., 1992, Reproductive biology of the planktonic copepod Paracalanus $s p$ in the inland sea of Japan. $J$. Plankton Res., 14:343-358.

WILLIS, K. J. \& LING, N., 2003, The toxicity of emamectin benzoate, an aquaculture pesticide, to planktonic marine copepods. Aquaculture, 221:289-297. doi: 10.1016/S00448486(03)00066-8 